\title{
ANÁLISE GENÉTICA DE CARACTERÍSTICAS REPRODUTIVAS NA RAÇA BRAHMAN
}

\author{
GENETIC ANALYSIS OF REPRODUCTIVE TRAITS INBRAHMAN CATTLE
}

\author{
Faria, L.C. ${ }^{1}$, Queiroz, S.A. ${ }^{2 A}$, Lôbo, R.B. ${ }^{3}$, Buzanskas, M.E. ${ }^{2 B}$, Venturini, G.C. ${ }^{2 C}$, Munari, D.P. ${ }^{2 D}$ \\ e Oliveira, J.A. ${ }^{2 E}$
}

\begin{abstract}
'Associação dos Criadores de Brahman do Brasil. Uberaba, MG. Brasil. lydiocf@yahoo.com.br ${ }^{2}$ Universidade Estadual Paulista (UNESP). Faculdade de Ciências Agrárias e Veterinárias (FCAV).

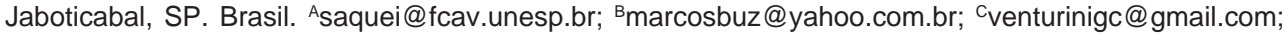
Ddanisio@fcav.unesp.br; Ejaoliv@fcav.unesp.br

${ }^{3}$ Associação Nacional de Criadores e Pesquisadores. Ribeirão Preto, SP. Brasil. ancp@ancp.org.br
\end{abstract}

\section{PaLAVRAS ChaVE ADICIONAIS}

Eficiência relativa de seleção. Parâmetros genéticos. Perímetro escrotal. Período de gestação. Peso ao nascer.

\section{RESUMO}

Para fins de estabelecer critérios de seleção para a raça Brahman, no Brasil, este estudo objetivou estimar parâmetros genéticos e ambiental para peso ao nascer (PN), período de gestação (PG) e perímetro escrotal aos 365 (PE365), 455 (PE455) e 550 (PE550) dias de idade e eficiências relativas de seleção indireta versus a direta para essas características. Os dados referem-se a 15851 animais de 16 rebanhos de seis estados, participantes de um programa de melhoramento genético. Componentes de (co)variância e coeficientes de herdabilidade e de correlações genéticas e ambientais entre características foram estimados pelo método da máxima verossimilhança restrita, sob modelo animal multi-característica. No modelo, foram incluídos como efeitos fixos, grupo de contemporâneos e idade da vaca ao parto como covariável (efeitos linear e quadrático) e, como efeitos aleatórios, genético aditivo direto, ambiente permanente da vaca e resíduo. Para PN e PG considerou-se ainda o efeito aleatório genético materno. As herdabilidades estimadas para PN, PG, PE365, PE455 e PE550 foram, respectivamente, 0,$36 ; 0,29 ; 0,55 ; 0,43$ e 0,40 . As correlações genéticas entre PN e PG, PGe PE365, PG e PE455, PG e PE550, PE365 e PE455, PE365 e PE550 e PE455 e PE550 foram, respectivamente, 0,06; 0,$13 ; 0,20 ; 0,13 ; 0,96 ; 0,98$ e 0,99 . Todas as

\section{AdDitiONAL KEYWORDS}

Birth weight. Genetic parameters. Gestation length. Relative efficiency of selection. Scrotal circumference.

características apresentaram variabilidade genética aditiva suficiente para responder favoravelmente à seleção. No que tange a precocidade sexual, a melhor opção seria PE365, devido à alta herdabilidade estimada, além de que a seleção indireta a esta idade seria mais eficiente que a direta para PE455.

\section{SUMMARY}

Aiming to establish selection criteria for the Brahman cattle in Brazil, the objective of this paper was to estimate genetic and environmental parameters for birth weight (BW), gestation length (GL) and scrotal circumference at 365 (SC365), 455 (SC455) and 550 (SC 550) days of age, and relative efficiency of indirect versus direct selection for these traits. Data were obtained from 15851 animals belonging to 16 herds in six states, enrolled in a genetic breeding program. (Co)variance and heritability coefficients and genetic and environmental correlations between traits were estimated by restricted maximum likelihood (REML) under a multi trait animal model. The model included as fixed effects the contemporary group and the age of cow at calving as a covariate (linear and quadratic), and as random the direct genetic, the permanent environmental and residual effects. For BW and 
GL it was also considered the maternal genetic effect as random. Estimates of direct heritabilities were $0.36,0.29,0.55,0.43$ and 0.40 for $B W, G L$, SC365, SC455 and SC550, respectively. Genetic correlations between BW and GL, GL and SC365, GL and SC455, GL and SC550, SC365 and SC455, SC365 and SC550 and SC455 and SC550, were respectively $0.06,0.13,0.20,0.13,0.96,0.98$ and 0.99 . The heritabilities estimates for all traits indicated these show enough additive genetic variability to respond favorably to selection. For sexual precocity, the best option would be SC365, due to the high value of heritability and to indirect selection at this age being more efficient than direct selection for PE455.

\section{INTRODUÇÃO}

Os problemas reprodutivos são os principais limitantes da eficiência produtiva em bovinos de corte (Donoghue, 2006) e, por isso, a inclusão de características reprodutivas nos objetivos de seleção seria indispensável para a maximização da eficiência econômica dos rebanhos (Queiroz et al., 2005). Apesar disso, os programas mais tradicionais de melhoramento genético têm dado ênfase quase exclusivamente às características de desempenho ponderal.

Em vários programas de melhoramento de bovinos de corte no Brasil (Lôbo et al., 2011, CRV Lagoa, 2011), a fertilidade dos machos vem sendo incluída como critério de seleção para aumentar a taxa de natalidade imediata e permanente, uma vez que a baixa herdabilidade das características de fertilidade das fêmeas aliada à baixa intensidade de seleção, normalmente aplicada a esse sexo, resultam em baixo progresso genético esperado.

O perímetro escrotal apresenta herdabilidade de moderada à alta magnitude e tem sido utilizado como um indicador da precocidade sexual de machos. Esta característica também apresenta correlação genética negativa com características reprodutivas, tais como, idade ao primeiro parto (Barrozo et al., 2012), dias para o parto e período de gestação (Grupioni, 2012).
Desta forma, seleção para perímetro escrotal implicaria em ganhos genéticos para essas características de eficiência reprodutiva em fêmeas.

O período de gestação é também uma importante característica relacionada com a reprodução de bovinos, uma vez que vacas com menores períodos de gestação têm vantagem reprodutiva sobre as de gestação mais longa (Rocha et al., 2005). Rocha et al. (2005) e Grupioni (2012) estimaram os valores 0,21 e 0,48 para a herdabilidade dessa característica em bovinos Nelore e Guzerá, respectivamente. Por outro lado, as estimativas para perímetro escrotal em várias idades variam de 0,29 a 0,65 (Yokoo et al., 2007; Frizzas et al., 2009; Grossi et al., 2009; Barrozo et al., 2012 e Grupioni, 2012). Vargas et al. (1998) estimaram em 0,28 a herdabilidade para o perímetro escrotal aos 18 meses de idade em bovinos da raça Brahman. As estimativas de correlações genéticas entre o perímetro escrotal em várias idades são de magnitude alta, acima de 0,68 , sendo maiores quando avaliadas entre idades próximas (Yokoo et al., 2007 e Frizzas et al., 2009).

A raça bovina Brahman teve sua origem no século XIX, nos Estados Unidos da América, a partir do cruzamento entre indivíduos das raças Nelore, Gir, Guzerá e Krishna Valley (Faria et al., 2010). No Brasil, sua introdução ocorreu no ano de 1994, com a importação de animais originários desse país, e posteriormente, da Argentina, Colômbia e Paraguai, totalizando 976 animais vivos que aqui ingressaram até abril de 1994 (ABCZ, 2010). Desde sua introdução, a raça está em crescimento, o que pode ser verificado por meio de dados estatísticos da Associação Brasileira dos Criadores de Zebu (ABCZ). No período de 1995 a 2009, o volume de registros genealógicos de nascimento aumentou em média, $129 \%$ ao ano e o total de animais com registro definitivo nesse período é de cerca de 64000 indivíduos. A expansão da raça Brahman vem sendo feita, principalmente, por meio do uso de sêmen, 


\section{ANÁLISE GENÉTICA DE CARACTERÍSTICAS REPRODUTIVASNA RAÇA BRAHMAN}

fertilização in vitro e transferências de embriões (ABCZ, 2010). No entanto, devido a sua recente introdução no Brasil, poucos estudos foram efetuados com o intuito de subsidiar a elaboração de diretrizes ao programa de melhoramento da raça.

Assim, este estudo teve como objetivos estimar herdabilidades e correlações genéticas e de ambiente para o peso ao nascer $(\mathrm{PN})$, período de gestação $(\mathrm{PG})$ e perímetro escrotal aos 365 (PE365), 455 (PE455) e 550 (PE550) dias de idade e eficiências relativas de seleção indireta em comparação com a direta entre essas características, em bovinos Brahman, no Brasil, com o propósito de estabelecer critérios de seleção para esta raça.

\section{MATERIALE MÉTODOS}

$\mathrm{O}$ arquivo de dados analisado continha registros de 15851 animais participantes do Programa de Melhoramento Genético da Raça Brahman (PMGRB), sob gestão da Associação Nacional de Criadores e Pesquisadores (ANCP), Ribeirão Preto, SP, e referem-se a animais nascidos no período de 2000 a 2005, de 16 rebanhos localizados nos estados de São Paulo (SP), Minas Gerais (MG), Mato Grosso (MT), Mato Grosso do Sul (MS), Tocantins (TO) e Goiás (GO) (tabela I).

As fazendas participantes do programa tinham manejos diferenciados de acordo com as atividades realizadas em cada uma, pois alguns criadores participam de exposições e leilões de elite. Assim, alguns rebanhos foram manejados exclusivamente em pastagens, outros em pastagens e suplementados, ou em pastagens e confinados (tabela I). As pastagens mais comuns eram compostas por gramíneas dos gêneros Urochloa, Andropogon, Panicum e Cynodon. Alguns criadores adotaram técnicas de adubação e irrigação de pastagens, com pastejo rotacionado. Nos locais das fazendas ocorrem os tipos climáticos $\mathrm{Aw}$ e $\mathrm{Cw}$ de Köppen.
A maioria das fazendas adotou estação de acasalamentos, cuja duração variava de 60 a 90 dias, no período de outubro a março, conforme a região e o nível de manejo do rebanho. A utilização de técnicas de reprodução, como inseminação artificial (IA), fertilização in vitro (FIV) e transferência de embriões (TE), como também repasse de touros após a primeira ou segunda oportunidade de acasalamento, foram práticas comuns nos rebanhos analisados.

As informações dos animais foram submetidas à rigorosa averiguação, antes de serem incorporados ao arquivo de dados, e foram eliminadas aquelas discrepantes, biologicamente incorretas e às referentes às técnicas de reprodução FIV e TE.

Tabela I. Distribuição de bovinos da raça Brahman, de acordo com rebanho $(R)$, estado $(E)$ e tipo de manejo (TM).(Brahman cattle distribution, according to herd $(R)$, state $(E)$ and type of management (TM)).

\begin{tabular}{lccccc}
\hline E & R & № animais & \multicolumn{3}{c}{ No animais/TM } \\
& & & $P$ & $S$ & S \\
\hline GO & 1 & 75 & 23 & 30 & 22 \\
MG & 2 & 59 & 59 & 0 & 0 \\
MG & 3 & 1102 & 604 & 403 & 95 \\
MG & 4 & 64 & 54 & 2 & 8 \\
MS & 5 & 139 & 139 & 0 & 0 \\
MS & 6 & 552 & 543 & 9 & 0 \\
MS & 7 & 284 & 193 & 36 & 55 \\
MT & 8 & 3403 & 3200 & 155 & 48 \\
MT & 9 & 5134 & 4830 & 291 & 13 \\
SP & 10 & 35 & 35 & 0 & 0 \\
SP & 11 & 70 & 70 & 0 & 0 \\
SP & 12 & 926 & 670 & 177 & 79 \\
SP & 13 & 3193 & 2381 & 504 & 308 \\
SP & 14 & 420 & 52 & 268 & 100 \\
SP & 15 & 43 & 21 & 0 & 22 \\
TO & 16 & 352 & 280 & 8 & 64 \\
Total & 16 & 15851 & 13154 & 1883 & 814 \\
\hline
\end{tabular}

P:Pasto; SI:Semi-intensivo; I: Intensivo; GO: Goiás; MG: Minas Gerais; MS: Mato Grosso do Sul; MT: Mato Grosso; SP: São Paulo; TO: Tocantins. 
Um resumo descritivo das características estudadas, após a consistência dos dados, é apresentado na tabela II. O peso ao nascer e o período de gestação foram tomados como medidas repetidas da vaca. Os perímetros escrotais foram medidos em torno de 12,15 e 18 meses e ajustados para as idades padrão de 365 (PE365), 455 (PE455) e 550 (PE550) dias.

Os componentes de (co)variância e os coeficientes de herdabilidade e de correlações genética e de ambiente entre as características estudadas foram estimados por REML usando um algoritmo livre de derivada, sob modelo animal multi-característica. No modelo de período de gestação e peso ao nascer foram considerados, como efeitos fixos, grupo de contemporâneos (sexo do produto, rebanho, trimestre de parto, ano de parto e regime alimentar) e a (co)variável idade da vaca ao parto (efeitos linear e quadrático) e, como aleatórios, os efeitos genético direto, genético materno, de ambiente permanente não correlacionado e residual. O perímetro escrotal (PE) foi considerado como diferentes características nas três idades estudadas. O modelo de análise do PE incluiu o efeito de grupo de contemporâneos (rebanho, trimestre de nascimento, ano de nascimento e regime alimentar) como fixo e, os efeitos genético aditivo direto e residual, como aleatórios.
Grupos de contemporâneos com número de observações menores que três ou com filhos de um único pai foram eliminados.

O modelo animal multi-característica pode ser representado como:

$$
y=X b+Z_{1} u+Z_{2} m+Z_{3} p e+\varepsilon
$$

em que:

$y=0$ vetor de observações;

$b=0$ vetor de efeitos fixos;

$u=0$ vetor de efeitos genéticos aditivos diretos; $\mathrm{m}=0$ vetor de efeitos aditivos genéticos maternos; $\mathrm{pe}=0$ vetor dos efeitos de ambiente permanente materno;

$\varepsilon=0$ vetor de efeitos aleatórios residuais;

$X, Z_{1}, Z_{2}$ e $Z_{3}=$ são as matrizes de incidência que associam b, u, m e pe com y, respectivamente.

Foi assumido que:

$E[y]=X b$

$\operatorname{Var}(\mathrm{u})=\mathrm{G}_{\mathrm{d}} \otimes A$

$\operatorname{Var}(m)=G m \otimes A$

$\operatorname{Var}(\mathrm{pe})=\mathrm{P} \otimes{ }^{\prime}$

$\operatorname{Var}(\mathrm{e})=\mathrm{R} \otimes \mathrm{I}$

em que:

$\mathrm{G}_{\mathrm{d}}=$ matriz de (co)variâncias genética aditiva direta entre as características;

$A=$ matriz de parentesco, obtida com informações do pedigree de 20213 animais;

$\mathrm{G}_{\mathrm{m}}=$ matriz de (co)variâncias genética aditiva materna;

$\mathrm{P}=$ matriz de (co)variâncias dos efeitos de ambiente permanente do animal;

Tabela II. Número de observações $(N)$, médias, valores máximos e mínimos, desvios padrão $(D P)$ e coeficientes de variação $(C V)$ do peso ao nascer $(P N)$, período de gestação $(P G)$, perímetro escrotal aos 365 (PE365), 455 (PE455) e 550 (PE550) dias de idade de animais da raça Brahman. (Number of observations $(\mathrm{N})$, average, maximum and minimum values, standard deviations (DP) and coefficients of variation (CV) of birth weight (PN), gestation period (PG), scrotal circumference at 365 (PE365), 455 (PE455) and 550 (PE550) day-old animals of the Brahman breed).

\begin{tabular}{lcccccc}
\hline & N & Média & DP & Mínimo & Máximo & CV (\%) \\
\hline PN (kg) & 11085 & 33,03 & 4,13 & 16,00 & 60,00 & 12,49 \\
PG (dias) & 2707 & 290,32 & 10,66 & 245,00 & 315,00 & 3,67 \\
PE365 $(\mathrm{mm})$ & 1028 & 203,68 & 29,06 & 150,00 & 323,00 & 14,27 \\
PE455 $(\mathrm{mm})$ & 1216 & 229,09 & 32,98 & 150,00 & 361,00 & 14,40 \\
PE550 $(\mathrm{mm})$ & 473 & 250,00 & 25,03 & 171,00 & 364,00 & 10,01 \\
\hline
\end{tabular}

Archivos de zootecnia vol. 61, núm. 236, p. 562. 


\section{ANÁLISE GENÉTICA DE CARACTERÍSTICAS REPRODUTIVAS NA RAÇA BRAHMAN}

$\mathrm{R}=$ matriz de (co)variâncias para os efeitos aleatórios residuais;

I= matriz de identidade (de apropriada ordem);

$\otimes=$ operador do produto Kronecker.

O modelo acima foi ajustado usando o programa WOMBAT (Meyer, 2007), considerando que o critério de convergência foi alcançado quando a variância dos valores do logaritmo da função de verossimilhança foi igual ou menor que $10^{-9}$. Como havia possibilidade de se obterem máximos locais, em vez do máximo global (Press et al., 1986), várias reinicializações foram executadas no sentido de se assegurar a convergência no máximo global da função de verossimilhança.

A eficiência relativa $(G)$, em termos de ganho genético, da seleção praticada em uma característica sobre outra geneticamente correlacionada foi estimada pela fórmula apresentada por Faria et al. (2011):

Ganho genético em 2 sob seleção para 1

Ganho genético em 2 sob seleção para 2

que é igual ao produto da correlação genética entre características 1 e $2,\left(\mathrm{r}_{\mathrm{G}(1,2)}\right)$, pela raiz quadrada da razão entre as herdabilidades das mesmas $\left(\mathrm{h}_{1}^{2} \mathrm{e} \mathrm{h}_{2}^{2}\right.$, respectivamente), ou seja:

$$
G=r G(1,2) x \sqrt{h_{1}^{2} / h_{2}^{2}}
$$

em que:

se $G>1$, a seleção indireta é mais eficiente. Caso contrário, a seleção direta seria mais eficiente. O valor de G indica a proporção de redução ou aumento no ganho genético em relação à seleção direta.

\section{RESULTADOSEDISCUSSÃO}

As magnitudes das estimativas dos componentes de variância genética, fenotípica e residual, obtidas por análise multi-característica (tabela III), apresentaram valores crescentes em relação às características peso ao nascer (PN), período de gestação (PG), perímetro escrotal aos 365 (PE365), 455
(PE455) e 550 (PE550) dias de idade, nesta ordem. Esse resultado é esperado, pois tais medidas de variabilidade dependem da magnitude dos registros, de modo que, mesmo que essas características variassem igualmente em relação às suas respectivas médias, a variância de PN seria, mesmo assim, menor do que a variância das demais características, e assim sucessivamente, naquela ordem.

Com base nessas estimativas de componentes de variância, as estimativas de herdabilidade para o peso ao nascer, período de gestação e perímetros escrotais aos 365,455 e 550 dias de idades na raça estudada, variaram de 0,29 a 0,55 (tabela IV). A estimativa de herdabilidade obtida para o peso ao nascer foi igual a $0,36 \pm 0,04$ e evidencia que a característica apresenta variação aditiva suficiente para responder à seleção tanto para aumentar como para diminuir a média do peso ao nascimento. Entretanto, a seleção para aumentar o PN não é recomendada,

Tabela III. Estimativas de componentes de variância obtidas por análise multi-característica para o peso ao nascer $(P N)$, período de gestação $(P G)$, perímetro escrotal aos 365 (PE365), 455 (PE455) e 550 (PE550) dias de idade de animais da raça Brahman. (Estimates of variance components obtained by multi trait analysis for birth weight (PN), gestation period (PG), scrotal circumference at 365 (PE365), 455 (PE455) and 550 (PE550) day-old animals Brahman breed).

\begin{tabular}{lccccc}
\hline & $\hat{\sigma}_{\mathrm{g}}^{2}$ & $\hat{\sigma}_{\mathrm{e}}^{2}$ & $\hat{\sigma}_{\mathrm{f}}^{2}$ & $\hat{\sigma}_{\mathrm{m}}^{2}$ & $\hat{\sigma}_{\mathrm{pe}}^{2}$ \\
\hline PN & 4,07 & 6,09 & 10,97 & 0,46 & 0,35 \\
PG & 16,34 & 36,79 & 55,46 & 0,22 & 2,11 \\
PE365 & 189,11 & 152,26 & 342,07 & - & 0,70 \\
PE455 & 230,83 & 259,96 & 538,27 & - & 47,48 \\
PE550 & 250,80 & 354,68 & 618,60 & - & 13,12 \\
\hline
\end{tabular}

$\hat{\sigma}_{\mathrm{g}}^{2}$ : variância genética aditiva; $\hat{\sigma}_{\mathrm{e}}^{2}$ : variância residual; $\hat{\sigma}_{\mathrm{f}}^{2}$ : variância fenotípica; $\hat{\sigma}_{\mathrm{m}}^{2}$ : variância materna; $\hat{\sigma}_{\mathrm{pe}}^{2}$ : variância de ambiente permanente. 
FARIA, QUEIROZ, LÔBO, BUZANSKAS, VENTURINI, MUNARI E OLIVEIRA

Tabela IV. Estimativas de herdabilidade direta (na diagonal), correlações genéticas (acima da diagonal) e correlações de ambiente (abaixo da diagonal) e respectivos erros-padrão (entre parênteses), obtidas por análise multi-característica para o peso ao nascer (PN), período de gestação (PG), perímetro escrotal aos 365 (PE365), 455 (PE455) e 550 (PE550) dias de idade de animais da raça Brahman. (Estimates of direct heritability (diagonal), genetic correlations (above diagonal) and environmental correlations (below diagonal) and their standard errors (in parentheses) obtained by multi trait analysis for birth weight (PN), gestation period (PG), scrotal circumference at 365 (PE365), 455 (PE455) and 550 (PE550) day-old animals of the Brahman breed).

\begin{tabular}{lccccc}
\hline & PN & PG & PE365 & PE455 & PE550 \\
\hline PN & $\mathbf{0 , 3 6 ( 0 , 0 4 )}$ & $0,06(0,18)$ & $0,24(0,19)$ & $0,15(0,20)$ & $0,07(0,22)$ \\
PG & $0,07(0,06)$ & $\mathbf{0 , 2 9 ( 0 , 0 8 )}$ & $0,13(0,08)$ & $0,20(0,31)$ & $0,13(0,31)$ \\
PE365 & $0,12(0,11)$ & - & $\mathbf{0 , 5 5 ( 0 , 1 8 )}$ & $0,96(0,06)$ & $0,98(0,05)$ \\
PE455 & $0,02(0,10)$ & - & $0,72(0,07)$ & $\mathbf{0 , 4 3 ( 0 , 1 7 )}$ & $0,99(0,07)$ \\
PE550 & $0,07(0,10)$ & - & $0,49(0,13)$ & $0,77(0,06)$ & $\mathbf{0 , 4 0 ( 0 , 1 7 )}$ \\
\hline
\end{tabular}

pois poderia acarretar em aumento da incidência de partos distócicos. Deve-se procurar manter o $\mathrm{PN}$ próximo à média da raça, uma vez que bezerros que nascem leves têm maior probabilidade de vir a óbito. Boligon et al. (2009) relataram, para a raça Nelore, estimativa de herdabilidade igual a 0,25 , valor menor do que o obtido nesta pesquisa.

O período de gestação (PG) apresentou estimativa de herdabilidade de $0,29 \pm 0,08$, valor que também indica a possibilidade de se obter resposta à seleção. A seleção para menor $P G$ poderia minimizar problemas de parto e gerar menor impacto na taxa de crescimento, em comparação com o que seria esperado da seleção para menor peso ao nascimento. Além disso, reduziria o intervalo entre partos, embora essa redução deva provocar pequena alteração na eficiência reprodutiva do rebanho. A estimativa obtida para PG neste trabalho foi superior ao valor reportado por Rocha et al. (2005), para a raça Nelore $(0,21)$, mas menor do que o valor estimado por Grupione (2012), para a raça Guzerá $(0,48 \pm 0,11)$.

Em relação aos coeficientes de herdabilidade para o perímetro escrotal aos 365 (PE365), 455 dias (PE455) e 550 dias (PE4550) de idade, os valores estimados de $0,55 \pm 0,18$;
$0,43 \pm 0,17$ e $0,40 \pm 0,17$, respectivamente (tabela IV), evidenciam que a seleção para perímetro escrotal poderia proporcionar ganhos genéticos importantes para o mesmo. As referidas estimativas foram similares às relatadas por Yokoo et al. (2007) de, respectivamente, 0,$48 ; 0,53$ e 0,42 , enquanto que Grossi et al. (2009) reportaram herdabilidades para PE365 e PE450, iguais à, respectivamente, $0,48 \pm 0,07$ e $0,65 \pm 0,07$, em bovinos da raça Nelore. Porém, a estimativa para PE550 foi superior à citada por Vargas et al. (1998) para a raça $\operatorname{Brahman}(0,28)$.

A correlação genética estimada $(0,06 \pm$ 0,18 ) entre o peso ao nascer e o período de gestação (tabela IV) foi inferior à relatada por Bourdon e Brinks (1982) de 0,25, para bovinos das raças Red Angus, Angus e Hereford. A baixa magnitude da estimativa obtida neste estudo sugere que a seleção com base no PG não deve ter influência no peso ao nascer. No entanto, ao contrário, Lôbo et al. (2000) sugeriram que bezerros com gestação mais curta podem nascer mais leves. Por outro lado, Bourdon e Brinks (1982) colocaram em dúvida a eficácia do período de gestação como critério de seleção, sugerindo que seleção para crescimento, associada à seleção para menor duração de gestação, seria menos eficaz que seleção 
para maior taxa de crescimento e menor peso ao nascer, pois poderia tanto diminuir a duração de gestação como alterar a curva de crescimento.

As estimativas das correlações genéticas entre o período de gestação (PG) com o perímetro escrotal aos 365 (PE365), 455 (PE455) e 550 (PE550) dias de idade, foram, respectivamente, $0,13 \pm 0,08 ; 0,20 \pm 0,31$ e $0,13 \pm 0,31$ (tabela IV). Esses valores foram superiores às estimadas entre PG e PE550 por Pereira et al. (2000) e Pereira et al. (2002), de, respectivamente, $-0,05$ e 0,02 , na raça Nelore e por Grupione (2012), de -0,01 $\pm 0,15$ e 0,09 $\pm 0,16$, para PG ePE365 ePG ePE450, na raça Guzerá. A partir destes resultados, sugere-se que as características PG e PE praticamente não são influenciadas pelos mesmos genes. Portanto, a seleção com base em uma delas não deverá proporcionar resposta correlacionada na outra.

A correlação genética entre perímetro escrotal e peso ao nascer, de acordo com a revisão elaborada por Oliveira et al. (2007), varia de 0,10 a 0,22 , valores estes próximos às estimativas obtidas no presente estudo, as quais variaram de 0,07 a 0,24 (tabela IV). Segundo esses autores, deve-se dar atenção à correlação entre essas características, que em muitos rebanhos é desfavorável, pois ao se aumentar o perímetro escrotal, pode aumentar o peso ao nascer dos bezerros e, como conseqüência, as distocias.

As estimativas das correlações genéticas obtidas entre PE365, PE455 e PE550, de 0,96 a 0,99 (tabela IV), sugerem que os perímetros escrotais às idades estudadas podem ser considerados em termos genéticos como sendo a mesma característica. Assim, podese adotar como critério de seleção para perímetro escrotal nos machos, visando identificar animais mais precoces sexualmente, $\mathrm{o}$ valor medido aos 365 dias de idade, em razão da antecipação da medida e também pelo maior valor da estimativa da herdabilidade. Na raça Nelore, estimativas de correlações genéticas pouco inferiores às obtidas nesse estudo foram relatadas por Yokoo et al.
(2007), que variaram de 0,76 a 0,96 , por Frizzas et al. (2009), cuja estimativa $(0,89)$ foi obtida entre os perímetros tomados aos 12 e 18 meses de idade.

Quanto às estimativas de correlações de ambiente (tabela IV) verifica-se que são positivas e, exceto as envolvendo $\mathrm{PN}$, de média a alta magnitude $(0,49 \pm 0,13$ a $0,72 \pm 0,07)$. Este resultado sugere que, principalmente os perímetros escrotais, são influenciados, no mesmo sentido, pelas mesmas diferenças de condições de ambiente e efeitos genéticos não aditivos.

Convém mencionar que os erros-padrão das estimativas das correlações genéticas e de ambiente entre as características analisadas (tabela IV), exceto entre os perímetros escrotais nas diferentes idades, foram de grande magnitude, sugerindo baixa precisão das estimativas.

A revisão elaborada por Oliveira et al. (2007) e a pesquisa de Barrozo et al. (2012) relacionaram estimativas de correlações genéticas entre perímetro escrotal (PE) e idade ao primeiro parto (IPP) que variam de $-0,22$ a $-1,0$. De acordo com esses trabalhos a herdabilidade de PE (de 0,31 a 0,71$)$ é maior do que de IPP (de 0,01 a 0,39) e sendo correlacionado favoravelmente com idade ao primeiro parto, podem-se obter resultados convenientes ao selecionar indiretamente IPP. Por isso, a inclusão de PE é indicada em programas que visem melhorar a eficiência reprodutiva.

As estimativas das eficiências relativas (G) em termos de ganho genético de seleção indireta (2) versus seleção direta (1) PE365 (1) vs. PE455 (2), PE365 (1) vs. PE550 (2) $\mathrm{e}$ PE455 (1) vs. PE550 (2) foram, respectivamente, 1,$09 ; 1,15$ e 1,03 . As estimativas de eficiências relativas entre PG e PN com as demais características não foram consideradas devido aos altos erros-padrão das correlações genéticas obtidas nessa pesquisa.

Assim, quando o objetivo for a seleção com base no perímetro escrotal, verifica-se que seleção direta para maior perímetro aos 


\section{FARIA, QUEIROZ, LÔBO, BUZANSKAS, VENTURINI, MUNARI E OLIVEIRA}

365 dias de idade seria mais eficiente sobre PE455 (G=1,09) ePE550 $(\mathrm{G}=1,15)$ do que a seleção direta para essas características. Este resultado pode ser explicado pelo fato que essas características possuem altas correlações genéticas, sendo que a estimativa de herdabilidade de PE365 é suficientemente maior do que as de PE455 e PE550, para produzir, sob seleção em PE365, uma resposta correlacionada em PE455 e PE550 maior do que a direta nas mesmas. Comparando-se a seleção indireta em PE550 com a direta aos 455 dias de idade $(\mathrm{G}=1,03)$, verifica-se que a seleção indireta em PE550, sob seleção direta em PE455, seria mais eficiente que a direta em PE550. Recomenda-se então para a raça Brahman, por razões econômicas de tempo e espaço, se a seleção for baseada no perímetro escrotal, que esta seja feita aos 365 dias de idade do animal.

\section{BIBLIOGRAFIA}

ABCZ. 2010. Associação Brasileira de Criadores de Zebu. Uberaba. <http://www.abcz. org.br/ conteudo/tecnica/estatisticas.html> (26/04/ 2011).

Barrozo, D., Buzanskas, M.E., Oliveira, J.A., Munari, D.P., Neves, H.H.R. and Queiroz, S.A. 2012. Genetic parameters and environmental effects on temperament score and reproductive traits of Nellore cattle. Animal, 6: 36-40.

Boligon, A.A., Albuquerque, L.G., Mercadante, M.E.Z. e Lôbo, R.B. 2009. Herdabilidades e correlações entre pesos do nascimento à idade adulta em rebanhos da raça Nelore. Rev Bras Zootecn, 38: 2320-2326.

Bourdon, R.M. and Brinks, J.S. 1982. Genetic, environmental and phenotypic among gestation length, birth, growth traits and age at first calving in beef cattle. J Anim Sci, 55: 543-553.

CRV Lagoa. 2011. Sumário PAINT consolidado. http://www.crvlagoa.com.br/paint.asp?Op=2 (14/3/2012).

Donoghue, K.A. 2006. Genetic evaluation of female reproductive performance. http://www.bifcon ference.com/bif2002/Baker_Essay_pdfs/ Donoghue_02BIF.pdf (02/04/2006).

Faria, L.C., Queiroz, S.A., Vozzi, P.A., Lôbo, R.B.,

\section{CONCLUSÕES}

O peso ao nascer, o período de gestação (PG) e o perímetro escrotal em bovinos da raça Brahman, no Brasil, podem responder à seleção, sendo que a utilização do menor PG como critério de seleção, pode levar a uma leve diminuição no peso ao nascer (PN), mas, em proporção menor do que a seleção direta para menor PN. O perímetro escrotal aos 365 dias de idade pode ser adotado como critério de seleção para melhorar as características reprodutivas dos machos.

\section{AGRADECIMENTOS}

À ANCP pela sessão dos dados e ao CNPq e a Capes pela concessão de bolsa em produtividade em pesquisa e de estudo aos autores.

Magnabosco, C.U. e Oliveira, J.A. 2010. Variabilidade genética da raça Brahman no Brasil detectada por meio de análise de pedigree. Pesqui Agropecu Bras, 45: 1133-1140.

Faria, L.C., Queiroz, S.A., Vozzi, P.A., Lôbo, R.B., Bezerra, L.A.F., Magnabosco, C.U. and Oliveira, J.A. 2011. Quantitative genetic study on growth traits of Brahman cattle in Brazil. Ars Vet, 27:3035.

Frizzas, O.G., Grossi, D.A., Buzanskas, M.E., Paz, C.C.P., Bezerra, L.F., Lôbo, R.B., Oliveira, J.A. and Munari, D.P. 2009. Heritability estimates and correlations for body weight and scrotal circumference adjusted to 12 and 18 months of age for male Nellore cattle. Animal, 3: 347-351. Grossi, D.A., Venturini, G.C., Paz, C.C.P., Bezerra, L.A.F., Lôbo, R.B., Oliveira, J.A. and Munari, D.P. 2009. Genetic associations between age at first calving and heifer body weight and scrotal circumference in Nelore cattle. J Anim Breed Genet, 126: 387-93.

Grupioni, N.V. 2012. Associações genéticas entre características reprodutivas e de crescimento em bovinos da raça Guzerá. Jaboticabal (SP). Dissertação de Mestrado (Curso de PósGraduação em Genética e M Melhoramento 


\section{ANÁLISE GENÉTICA DE CARACTERÍSTICAS REPRODUTIVAS NA RAÇA BRAHMAN}

Animal). Faculdade de Ciências Agrárias e Veterinárias. Universidade Estadual Paulista 'Júlio de Mesquita Filho'. 41 f.

Lôbo, R.N.B., Madalena, F.E. and Vieira, A.R. 2000. Average estimates of genetic parameters for beef and dairy cattle in tropical regions. Anim Breed Abst, 68: 433-462.

Lôbo, R.B., Bezerra, L.A.F., Vozzi, P.A., Magnabosco, C.U., Albuquerque, L.G., Sainz, R.D., Bergmann, J.A.G., Faria, C.U. e Oliveira, H.N. 2011. Avaliação Genética de Touros da Raça Nelore, Guzerá, Brahman e Tabapuã. Sumário 2011. Associação Nacional de Criadores e Pesquisadores. Ribeirão Preto. 136 pp.

Meyer, K. 2007. WOMBAT-A tool for mixed model analysis in quantitative genetics by restricted maximum likelihood (REML). J Zhejiang Univ Sci B, 8: 815-821.

Oliveira, M.M., Rota, E.L., Dionello, N.J.L. e Aita, M.F. 2007. Herdabilidade e correlações genéticas do perímetro escrotal e idade ao primeiro parto com características produtivas em bovinos de corte: revisão. Rev Bras Agroc, 13: 141-146.

Pereira, E., Eler, J.P. e Ferraz, J.B.S. 2000. Correlação genética entre perímetro escrotal $e$ algumas características reprodutivas na raça Nelore. Rev Bras Zootecn, 29: 1676-1683.
Pereira, E., Eler, J.P. e Ferraz, J.B.S. 2002. Análise genética de características reprodutivas na raça Nelore. Pesqui Agropecu Bras, 37: 703708.

Press, W.H., Flannery, B.P., Teukolsky, S.A. and Vetterling, W.T. 1986. Numerical recipes. Cambridge University Press. Cambridge. 818 $\mathrm{pp}$

Queiroz, S.A., Pelicioni, L.C., Silva, B.F., Sesana, J.C., Martins, M.I.E.G. e Sanches, A. 2005. Índices de seleção para um rebanho Caracu de duplo propósito. Rev Bras Zootecn, 34: 827-837.

Rocha, J.C.M.C., Tonhati, H., Alencar, M.M. e Lôbo, R.B. 2005. Componentes de variância para o período de gestação em bovinos de corte. Arq Bras Med Vet Zoo, 57: 784-791.

Vargas, C.A., Elzo, M.A., Chase, J.R., Chenoweth, P.J. and Olson, T.A. 1998. Estimation of genetic parameters for scrotal circumference, age at puberty in heifers, and hip height in Brahman cattle. J Anim Sci, 76: 2536-2541.

Yokoo, M.J.I., Albuquerque, L.G., Lôbo, R.B., Sainz, R.D., Carneiro Junior, J.M., Bezerra, L.A. e Cunha Araujo, F.R. 2007. Estimativas de parâmetros genéticos para altura do posterior, peso e circunferência escrotal em bovinos da raça Nelore. Rev Bras Zootecn, 36: 1761-1766. 